PNL-SA-24177

\title{
GAMMA SPECTRAL ANALYSIS VIA NEURAL NETWORKS
}

P. E. Keller

R. T. Kouzes

October 1994

Presented at the

Nuclear Science Symposium Conference

October 30 - November 5, 1994

Norfolk, Virginia

Prepared for

the U.S. Department of Energy

under Contract DE-AC06-76RLO 1830

Pacific Northwest Laboratory

Richland, Washington 99352

\section{DISCLAIMER}

This report was prepared as an account of work sponsored by an agency of the United States Government. Neither the United States Government nor any agency thereof, nor any of their employees, makes any warranty, express or implied, or assumes any legal liability or responsibility for the accuracy, completeness, or usefulness of any information, apparatus, product, or process disclosed, or represents that its use would not infringe privately owned rights. Reference herein to any specific commercial product, process, or service by trade name, trademark, manufacturer, or otherwise does not necessarily constitute or imply its endorsement, recommendation, or favoring by the United States Government or any agency thereof. The views and opinions of authors expressed herein do not necessarily state or reflect those of the United States Government or any agency thereof. 


\section{DISCLAIMER}

Portions of this document may be illegible in electronic image products. Images are produced from the best available original document. 


\title{
Gamma Spectral Analysis via Neural Networks
}

\author{
Paul E. Keller and Richard T. Kouzes 1 \\ Environmental Molecular Sciences Laboratory, Pacific Northwest Laboratory, K1-87, P.O. Box 999, Richland, WA 99352, USA \\ pe_keller@pnl.gov and rt_kouzes@pni.gov
}

\begin{abstract}
A system combining a portable gamma-ray spectrometer with a neural network is discussed. In this system, the neural network is used to automatically identify radioactive isotopes in real-time from their gamma-ray spectra. Two neural network paradigms are examined: the linear perceptron and the optimal linear associative memory (OLAM). A comparison of the two paradigms shows that OLAM is superior to linear perceptron for this application. Both networks have a linear response and are useful in determining the composition of an unknown sample when the spectrum of the unknown is a linear superposition of known spectra. One feature of this technique is that it uses the whole spectrum in the identification process instead of only the individual photo-peaks. For this reason, it is potentially more useful for processing data from lower resolution gamma-ray spectrometers. This approach has been successfully tested with data generated by Monte Carlo simulations and with field data from both sodium iodide and germanium detectors. With the neural network approach, the intense computation takes place during the training process. Once the network is trained, normal operation consists of propagating the data through the network, which results in rapid identification of samples in the field. This approach is useful in situations that require fast response but where precise quantification is less important.
\end{abstract}

\section{INTRODUCTION}

Enormous amounts of hazardous waste were generated by more than 40 years of plutonium production at the U.S. Department of Energy's Hanford site. There are an estimated 1700 waste sites distributed around the 1450 square kilometers of southeastern Washington state that comprise this site [1]. This waste includes nuclear waste (e.g., fission products), toxic chemical waste (e.g., carbon tetrachloride, ferrocyanide, nitrates, etc.), and mixed waste (combined radioactive and chemical waste). The Pacific Northwest Laboratory is exploring the technologies required to perform environmental restoration and waste management in a cost-effective manner. This includes the development of compact, portable, and inexpensive systems capable of real-time identification of contaminants in the field. The objective of our research is to demonstrate the potential information processing capabilities of the neural network paradigm in real-time, automated identification

\footnotetext{
IThis work was supported by the Laboratory Directed Research and Development program at Pacific Northwest Laboratory (PNL). PNL is a multiprogram national laboratory operated by Battelle Memorial Institute for the U.S. Department of Energy under Contract DEAC06-76RLO 1830.
}

of contaminants. In this paper, artificial neural networks (ANNs) that can be used with a portable gamma-ray spectrometer are presented.

\section{ARTIFICIAL NEURAL NETWORKS}

ANNs are used in a wide variety of data processing applications where real-time data analysis and information extraction are required. One advantage of the neural network approach is that most of the intense computation takes place during the training process. Once the ANN is trained for a particular task, operation is relatively fast and unknown samples can be rapidly identified in the field. An ANN coupled to a sensing system, such as a spectrometer, can be used as a portable, automated system for identifying contaminants.

The traditional approach to gamma-ray spectral analysis can be categorized as finding peaks and fitting curves. This approach involves an iterative process of spectrum decomposition and regeneration until a mathematically synthesized spectrum closely matching the true spectrum can be generated. This is both time consuming and often requires manual intervention. The ANN approach employs pattern recognition on the entire spectrum. This recognition is performed by a single vector-matrix multiplication that results in rapid, real-time identification of analytes and can be used in automated systems.

For a sample composed of a combination of isotopes, the spectrum of the sample, $\underline{S}$, is approximately a linear superposition of the spectra of each individual isotope, $\underline{s}$. This is illustrated by Equation 1 where $\alpha_{i}$ is the relative concentration of each isotope in the sample.

$$
\underline{S}=\sum_{i} \alpha_{i} \underline{s}_{i}
$$

Therefore, the classification system should have a linear response with respect to the input. This deviates from the majority of ANNs which implement a nonlinear response. However, even with a linear response, the ANN approach has advantages in speed and simplicity over traditional approaches and is useful in automated systems since isotopes can be identified without human intervention.

An ANN designed to have a linear response employs linear activation functions. A feedforward ANN that implements linear activation functions can be reduced to a network with a single input layer and single output layer. Therefore, the ANN used in this application has a single input and single output layer as illustrated in Figure 1. Two ANN paradigms were studied for implementing the linear response: the linear perceptron and the optimal linear associative memory. Both approaches to gamma-ray spectral analysis have been applied 
separately [2-5]. In this paper, these two ANN techniques are compared.

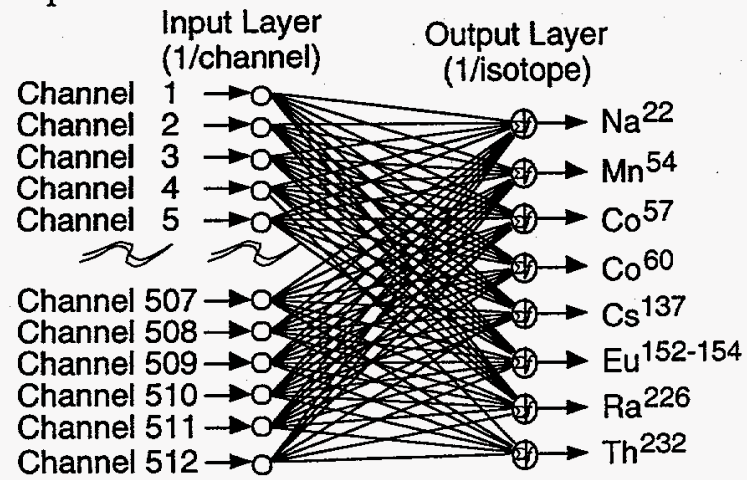

Fig. 1. An ANN used to identify radioactive isotopes.

\section{A. Linear Perceptron}

Linear perceptron is one of the oldest ANN paradigms. It originally sparked interest in the pattern recognition community in the late 1950s and early 1960s [6]. However, it was unable to solve pattern recognition problems that were not linearly separable. The original perceptron implemented a hardlimited threshold as the activation function. For the gammaray spectral analysis application, a modified linear activation function (linear for positive input and zero for negative input) known as a perceptron function is used. The delta rule is used to train the perceptron in an iterative process, which is detailed in Table I. With linear activation functions, this training algorithm is mathematically identical to the backpropagation algorithm [7] since the derivative terms in backpropagation would be unity in this case.

Table I

Perceptron Learning Rule

Step 1. Initialize weights with random values.

Step 2. Pick a labeled pattern (spectrum, $\underline{x}^{\mathrm{p}}$, and known composition, $\underline{t}^{\mathrm{p}}$ ) from the training set and present the spectrum to the network.

Step 3. Propagate data forward and generate the output classification.

$$
\underline{\underline{y}}^{\mathrm{p}}=\underline{\underline{\mathrm{W}}} \underline{\underline{x}}^{\mathrm{p}}
$$

Step 4. Calculate mean-square error between target classification and actual classification.

Step 5. Adapt the synaptic weights by using a delta rule to reduce output error.

$$
\Delta \underline{\underline{W}}=\eta\left(\underline{\underline{p}}^{\mathrm{p}}-\mathrm{y}^{\mathrm{p}}\right) \underline{x}^{\mathrm{p}} \quad(\eta=\text { learning rate })
$$

Step 6. If there are more spectra in the training set, loop back to step 2 .

Step 7. If the output error is high or the maximum number of iterations have not been met, then loop back to step 2 .

\section{B. Optimal Linear Associative Memory (OLAM)}

The optimal linear associative memory (OLAM) approach is based on a simple matrix associative memory model $[8,9]$. It was developed in the early 1970 s as a content addressable memory and is useful in situations where the input consists of a linear combination of known patterns (e.g., gamma-ray spectra). It is an improvement over the original matrix memory approach in that it projects an input pattern onto a set of orthogonal vectors where each orthogonal vector represents a unique pattern (exemplar). With linear activation functions, the training is a straight forward matrix orthogonalization process where each pattern from the training set is made to project onto a separate, unique orthogonal axis in the output space. This process is described in Table II.

Table II

OLAM Weight Specification

Step 1. Form matrices of spectra and isotopic concentrations. Arrange spectra, $\underline{x}^{p}$, as columns in an $n \times p$ dimensional matrix $\underline{\underline{X}}$ and target concentrations, $\underline{\mathrm{t}}^{\mathrm{p}}$, as columns in an $\mathrm{m} \times \mathrm{p}$ dimensional matrix $\underset{\mathrm{T}}{\mathrm{T}}$.

Step 2. Generate inverse of the spectral matrix X. Since $\underline{\underline{X}}$ is generally not a square matrix, a pseudoinverse technique is used to generate $\mathrm{X}^{\dagger}$. ( $\dagger$ indicates pseudo-inverse)

Step 3. Form the synaptic weight matrix.

$$
\underline{\underline{\mathrm{W}}}=\underline{\underline{\mathrm{TX}}}{ }^{\dagger}
$$

\section{PROTOTYPE SYSTEM LAYOUT}

Figure 2 illustrates a prototype system that combines a portable gamma-ray spectrometer with an ANN. Figure 1 illustrates the ANN that is connected to the gamma-ray spectrometer. In this prototype, a sodium iodide (NaI) detector is used, and 512 channels of data are produced by the spectrometer. All channels are fed into the ANN so that there is one input for every channel. There is a single processing layer (output layer) in the ANN where the number of output neurons is equal to the number of isotopes being identified ( 8 in this case). One feature of this approach to gamma-ray spectral analysis is that the whole spectrum is used in the identification process instead of individual peaks in the spectrum. For this

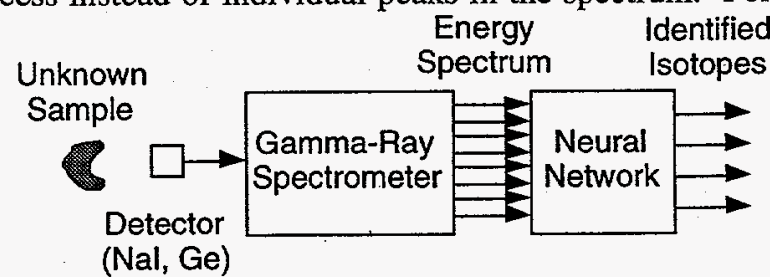

Fig. 2. Prototype system combining gamma-ray spectrometer with an ANN. 
reason, it is potentially more useful for processing data from lower resolution gamma-ray spectrometers like those employing NaI detectors.

Each isotope presented to the spectrometer produces a spectrum that is characteristic of that isotope. By presenting many different isotopes to the system, a database of spectra is constructed. From this database, training sets and test sets are generated. These sets are collections of labeled patterns (spectra of isotopes with known concentrations) representative of the desired identification mapping. The training sets are used to configure the ANN. The goal of this training is to learn an association between the spectra and the labels representing the spectra. ANNs were developed with both the linear perceptron learning rule and with the OLAM weight specification. The training process for the OLAM in a noniterative process, while the linear perceptron training process requires thousands of iterations. For this reason, it took only 200 milliseconds on a SPARCstation 10 to generate the OLAM ANN, while it took a couple of hours to generate the linear perceptron ANN. Training times on an Intel i 486 based personal computer are only a few times greater than this.

\section{RESULTS}

The prototype system illustrated in Figure 2 was tested with both Monte Carlo simulated spectra and field data collected from a gamma spectrometer equipped with a sodium iodide detector. Figure 1 illustrates the ANN configured to identify 8 radioactive isotopes $\left(\mathrm{Na}^{22}, \mathrm{Mn}^{54}, \mathrm{Co}^{57}, \mathrm{Co}^{60}\right.$, $\mathrm{Cs}^{137}, \mathrm{Eu}^{152-154}, \mathrm{Ra}^{226}$, and $\mathrm{Th}^{232}$ ) from their gamma-ray spectra. Field operation consists of presenting an unknown sample to the system, generating a gamma-ray spectrum, passing the spectrum through the ANN, and generating a classification of the unknown sample. The values on the output

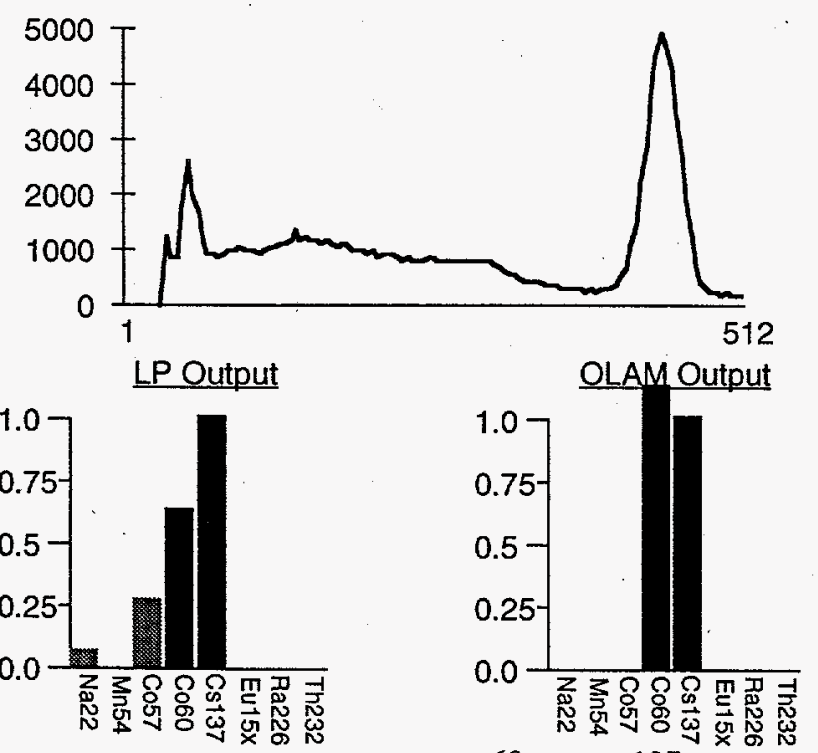

Fig. 3. Spectrum of a mixture of $\mathrm{Co}^{60}$ and Cs ${ }^{137}$ (sample 4) with the associated classifications by the linear perceptron (LP) and OLAM ANNs. ANN outputs in gray indicate incorrect identification of an isotope. neurons are proportional to the quantities of each radioactive isotope found in the sample. Figure 3 illustrates the classification of a sample composed of equal amounts of $\mathrm{Co}^{60}$ and $\mathrm{Cs}^{137}$ (sample 4). In this case, the OLAM correctly identified the composition of the samples while the linear perceptron incorrectly identified a significant amount of $\mathrm{Co}^{57}$. Figure 4 illustrates the classification of a sample composed of equal amounts of $\mathrm{Na}^{22}, \mathrm{Co}^{57}, \mathrm{Co}^{60}, \mathrm{Cs}^{137}$, and $\mathrm{Eu}^{152-154}$ (sample 5). In this case the OLAM correctly identifies all the isotopes present in the sample though the ratios between identified isotopes are not uniform. Figure 5 illustrates the classification

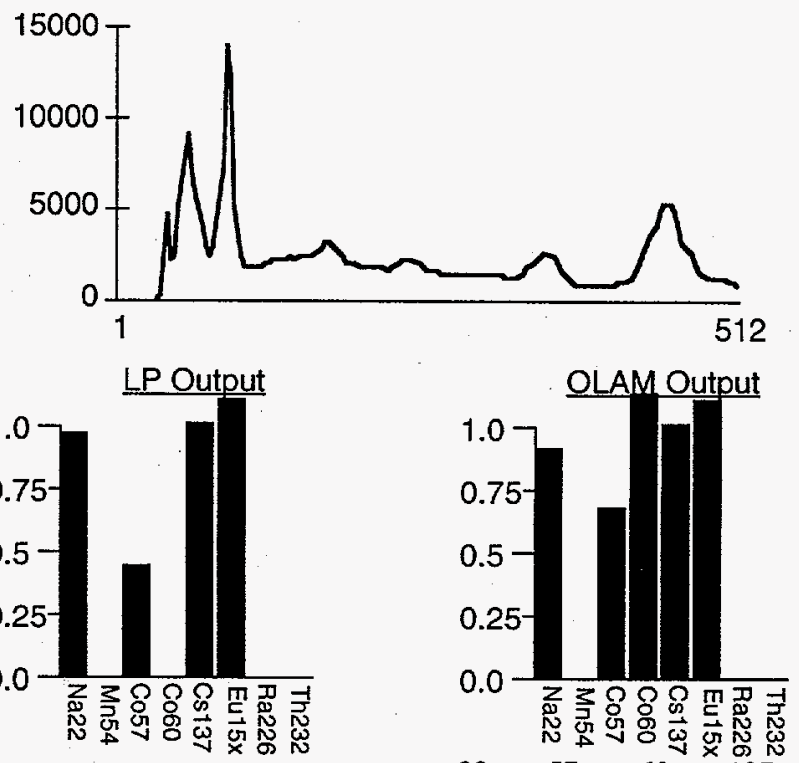

Fig. 4. Spectrum of a mixture of $\mathrm{Na}^{22}, \mathrm{Co}^{57}, \mathrm{Co}^{60}, \mathrm{Cs}^{137}$ and Eu 152-154 (sample 5) with the associated classifications by the linear perceptron (LP) and OLAM ANNs.
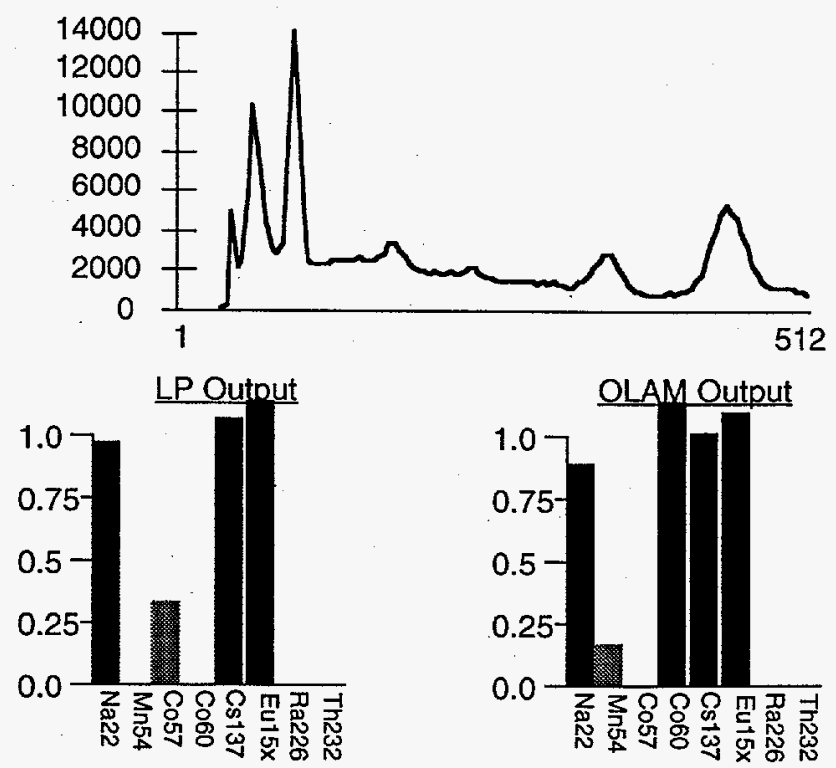

Fig. 5. Spectrum of a mixture of $\mathrm{Na}^{22}, \mathrm{Co}^{60}, \mathrm{Cs}^{137}$ and $\mathrm{Eu}^{152-154}$ (sample 7) with the associated classifications by the linear perceptron (LP) and the OLAM ANNs. ANN outputs in gray indicate incorrect identification of an isotope. 
Table III

Classification of actual spectra from $\mathrm{NaI}$ detector with linear perceptron (LP) and OLAM . Each column represents a different isotope. RMS Error is listed in the right-hand column.

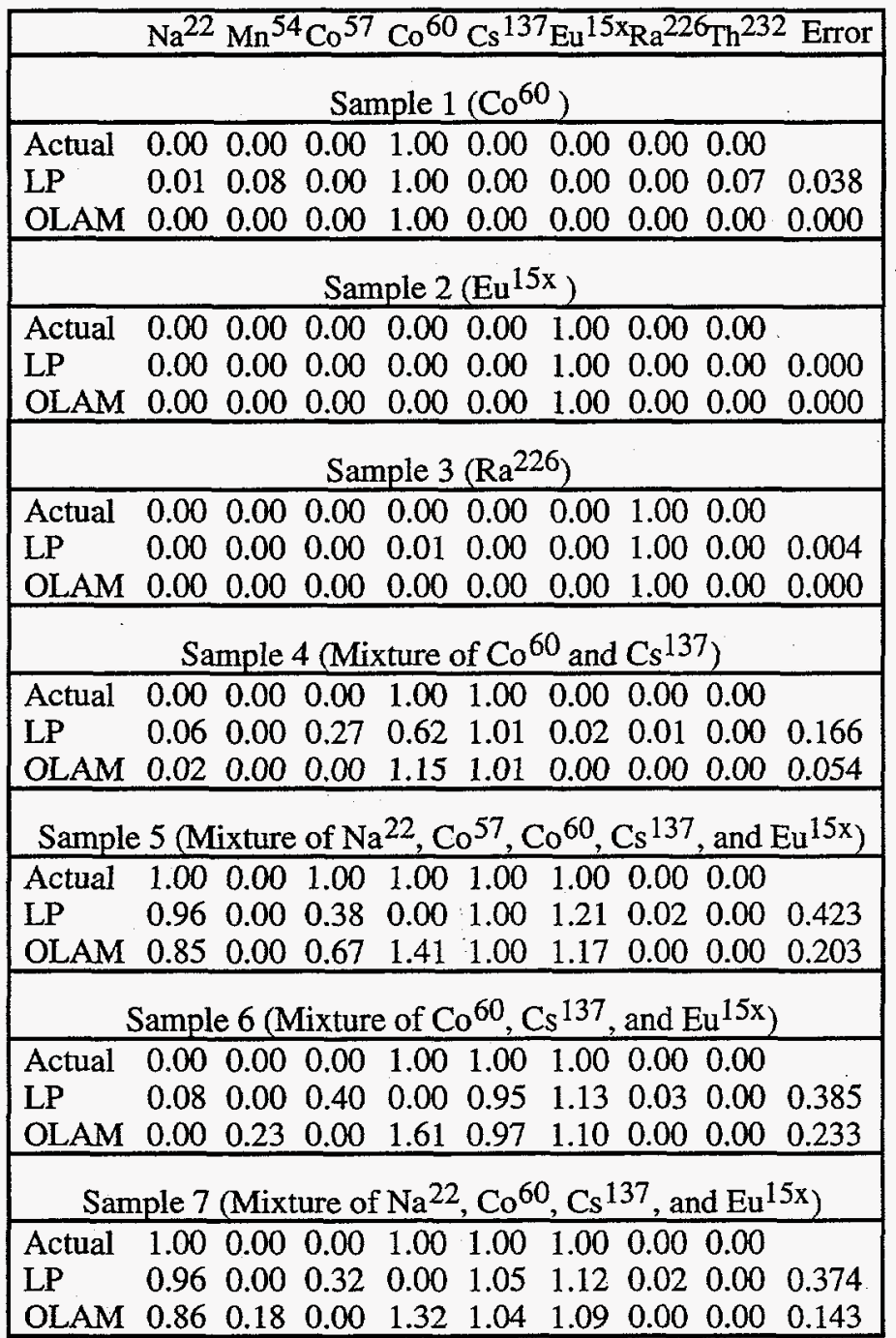

of a sample composed of equal amounts of $\mathrm{Na}^{22}, \mathrm{Co}^{60}, \mathrm{Cs}^{137}$ and $\mathrm{Eu}^{152-154}$ (sample 7). In this case, the OLAM incorrectly identified a small amount of $\mathrm{Mn}^{54}$ while the linear perceptron did not identify the presence of $\mathrm{Co}^{60}$ and misidentified a small a $\mathrm{Mn}^{54}$. The linear perceptron had a hard time identifying $\mathrm{Co}^{60}$ and often identified $\mathrm{Co}^{57}$ when it was not present. In several cases, the OLAM indicated a small amount of $\mathrm{Mn}^{54}$ when it was not present. However, the errors with the OLAM were always smaller than the linear perceptron. Similar results were found with other samples which can be found in Table III. Additional studies that were performed with a Germanium detector yielded similar results.

Table IV lists results from Monte Carlo simulated spectra. The linear perceptron and OLAM were tested on spectra of mixtures and on modified spectra. Spectra of mixtures were generated by combining the simulated spectra of the different isotopes. Modified spectra were produced by reducing the peak height of some spectra and by removing everything but
Table IV

Classification of Monte-Carlo generated spectra simulating a $\mathrm{NaI}$ detector with linear perceptron (LP) and OLAM ANNs. Each column represents a different isotope. RMS Error is listed in the right-hand column.

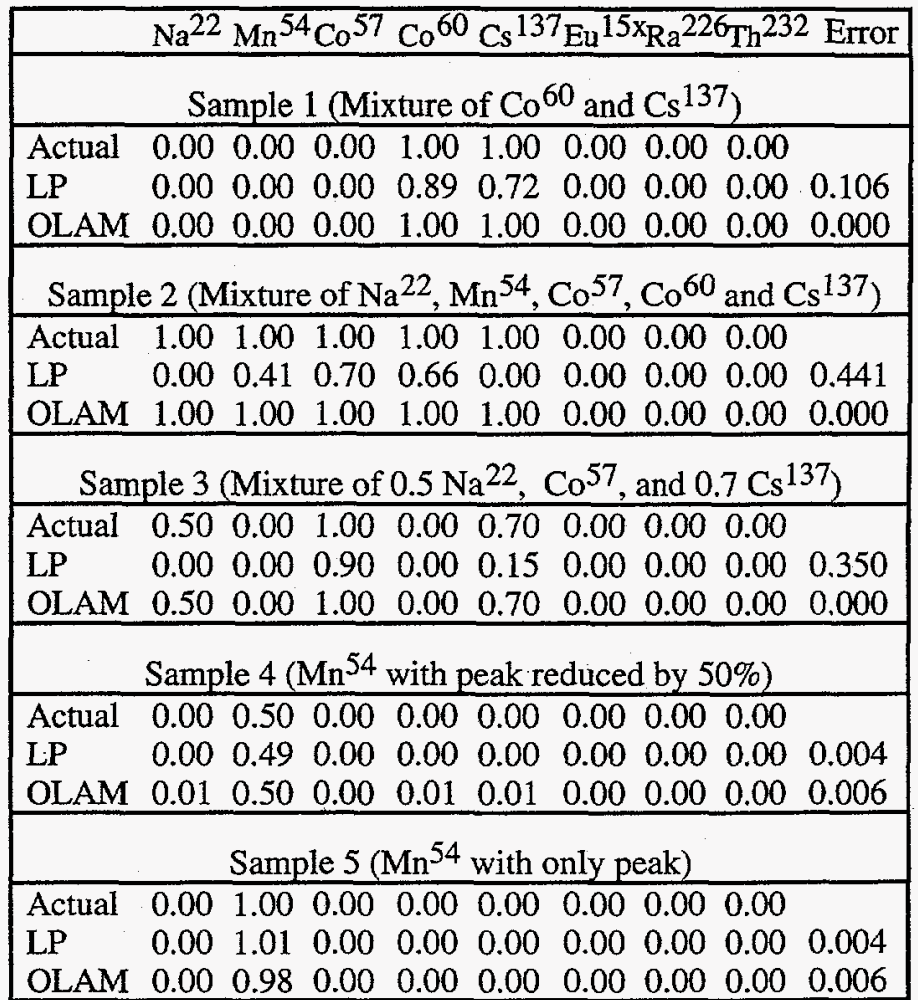

the peak of other spectra. Since both the spectrum of the mixtures and the OLAM were generated from ideal spectra, the OLAM perfectly identified all the isotopes in the different: mixtures. The linear perceptron did not fair well. For the modified spectra, the linear perceptron performed slightly better than the OLAM. These results indicate that the linear perceptron uses the peak information more than the OLAM does.

The time to identify an isotope from a 512 channel spectrum with 8 possible isotopes is 20 milliseconds on a $33 \mathrm{MHz}$ Intel i486DX based personal computer. Therefore, the classification process is limited not by the ANN but by the time it takes to acquire and generate a spectrum. In applications that can acquire and deliver data much faster, the ANN can be implemented in specialized hardware. In such a case, three orders of magnitude increase in classification speed can be achieved.

\section{DISCUSSION}

The initial results of our research have demonstrated the pattern recognition capabilities of the neural network paradigm in analyzing gamma-ray spectra. This study has shown the superior performance of the OLAM approach over the linear perceptron for gamma-ray spectral analysis in both classification accuracy and training speed. The classification performance can be attributed to the orthogonalization process used 
by the OLAM during training. Since this training process is non-iterative, the OLAM offers a substantially shorter training time than the linear perceptron.

One of the disadvantages of the OLAM, is that nearly ideal spectra are needed in the training process. However, if needed the OLAM can be provided with Monte Carlo generated spectra. The linear perceptron can be trained with noisy data or data with defects as long as a large training set is available.

This paper also illustrates some advantages of the ANN approach over conventional analytical techniques. These advantages include simplicity, real-time analysis, and automation. All of these are important in building compact and portable systems for automated contaminant identification. With this approach, the isotopes in a contaminant can be identified in a fraction of a second once the spectrum is sent to the ANN.

Further work could involve comparison of the ANN approach to more conventional techniques, exploration of other ANN paradigms, examination of techniques for combining ANN models, and development of field prototype systems. A field deployable system should work with different source geometries and should compensate for pile-up and gain shifting. An ANN that handles gain-shifting was recently reported by Olmos et. al. [10].

Information on ANN developments at Pacific Northwest Laboratory is available in the World Wide Web (WWW) pages of the Environmental Molecular Science Laboratory which is accessible through such WWW clients as NCSA Mosaic.

URL: http://www.emsl.pnl.gov:2080/docs/cie/neural/

\section{ACKNOWLEDGMENTS}

The authors wish to thank Brian Keele of Westinghouse Hanford Company for graciously providing gamma-ray spectra for this study.

\section{REFERENCES}

[1] B.G. Levi, "Hanford seeks short- and long-term solutions to its legacy of waste," Physics Today, vol. 45, no. 3, pp. 17-21, March 1992.

[2] P. Olmos, J.C. Diaz, J.M. Perez, P. Gomez, V. Rodellar, P. Aguayo, A. Bru, G. Garcia-Belmonte, and J.L. de Pablos, "A New Approach to Automatic Radiation Spectrum Analysis," IEEE Transactions on Nuclear Science, vol. 38, pp. 971-975, August 1991.

[3] P. Olmos, J.C. Diaz, J.M. Perez, G. Garcia-Belmonte, P. Gomez, V. Rodellar, "Application of neural network techniques in gamma spectroscopy," Nuclear Instruments and Methods in Physics Research, vol. A312, pp. 167-173, 1992.

[4] R. Koohi-Fayegh, S. Green, N.M.J. Crout, G.C. Taylor, and M.C. Scott, "Neural network unfolding of photon and neutron spectra using an NE-213 scintillation detector," Nuclear Instruments and Methods in Physics Research, vol. A329, pp. 269-276, 1993.

[5] P.E. Keller, R.T. Kouzes, and L.J. Kangas, "Applications of Neural Networks to Real-Time Data Processing at the Environmental and Molecular Sciences Laboratory," in
Conference Record of the Eighth Conference on Real-Time Computer Applications in Nuclear, Particle and Plasma Physics, Vancouver, BC, Canada, June 1993, pp. 438-440.

[6] F. Rosenblatt, "Two theorems of statistical separability in the Perceptron," in Mechanisation of Thought Process, Proceedings of symposium No. 10, National Physical Laboratory, London, November 1958, vol. I, pp. 421-456.

[7] D.E. Rumelhart, G.E. Hinton, and R.J. Williams, "Learning Internal Representations by Error Propagation," Parallel Distributed Processing Systems, Cambridge, MA: MIT Press 1986, vol. 1, pp. 318-362.

[8] T. Kohonen, "Correlation matrix memories," IEEE Transactions on Computers, vol. C-21, pp. 353, 1972.

[9] T. Kohonen, Self Organization and Associative Memory, third ed., New York: Springer-Verlag, 1989.

[10] P. Olmos, J.C. Diaz, J.M. Perez, P. Aguayo, P. Gomez, and V. Rodellar, "Drift Problems in the Automatic Analysis of GammaRay Spectra Using Associative Memory Algorithms," IEEE Transactions on Nuclear Science, vol. 41, no. 3, pp. 637-641, 1994. 